\title{
Ein Beitrag zur Morphologie der Reisblïte.
}

Von M. Akemine, Assistent-Professor am landwirtsehaftlichen Institut der Tohoku kaiserl. Universität zu Sapporo.

(Mit 5 Textabbildungen.)

Über die Morphologie der Reisblüte liegen bereits verschiedene Untersuchungen sowohl aus Europa als aus Japan vor. Ich selbst habe seit 1908 einige Beobachtungen darüber gemacht und schon im Jahre 1909 einen Teil der Resultate veröffentlicht'). In dem vorliegenden Aufsatze nun beabsichtige ich die Resultate meiner während vier Jahren (1908-1911) fortgesetzten Untersuchungen mitzuteilen, welche als ein Beitrag zur Pflanzenmorphologie einiges Interesse haben könnten. Die Sorte, welche ich als Untersuchungsmaterial auswählte, ist "Akage", die wichtigste Landsorte der kälteren Gegenden Japans. Um die Frage zu beantworten, $o b$ die unten beschriebenen Verbältnisse bei dieser Sorte auch für anderen Sorten zutreffen oder nicht, müssen noch weitere Untersuchungen abgewartet werden.

Bekanntlich besitzt jede Reisblüte zwei Blütenspelzen, nämlich Deckspelze (Palea inferior) und Vorspelze (Palea superior). An ibrer Basis
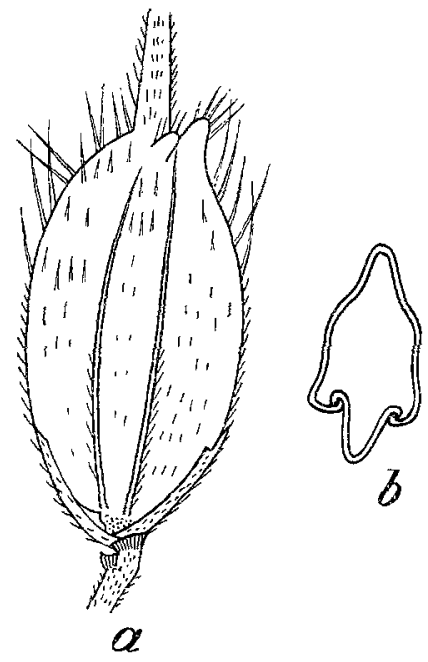

Abb. 1. $a$ Eine Reisblüte. $b$ Quer. schnitt der Spelzen. befinden sich zwei kurze Hüllspelzen, und darunter noch zwei winzige Hüllspelzen-Rudimente. Die ganze Oberfläche der Spelzen ist mit steifen Haaren bedeckt, welche nach der Spitze allmählich länger werden. Bemerkenswert ist die Struktur der Spitze der Blütenspelzen. Auf der Spize der Vorspelze befindet sich nämlich ein ziemlich großer Auswuchs, und auf derselben Stelle der Deckspelze sind zwei kleinere vorbanden, von denen je einer an beiden Seiten der Granne sitzt. Diese drei Auswüchse sind mit langen Haaren bedeckt. Die Ränder der Deck- und Vorspelze sind hakenförmig gekrümmt und dadurch miteinander sn fest verbunden, daß sie nicht leicht $z \mathfrak{a}$ trennen sind. Eine kleine Öffnung ist dabei an der Spitze vorhanden, durch welche vielleicht die Reizungen von außenher stattfinden dürften. Sowohl die eben erwähnten drei Auswächse als auch die darauf befindlichen Haare umschließen gerade diese Öffung, meines Erachtens um die Blüte vor dem Eintreten des Regenwassers zu schützen.

Zwischen den beiden Blätenspelzen findet man die eigentliche Blüte. Sie hat sechs Staubfäden, deren jeder an der Spitze einen Staubbeutel trägt. Er ist aus einem Paar von Pollensäcken zusammengesetzt,

1) M. Akemine, Studies on the Blooming and the Hand Pollination of Rice Plants. Journal of the Society of Agriculture and Forestry, Sapporo, 1909, Nos. $3-4$. 
welche am oberen Ende ein wenig und am unteren bis zu ca. 1/5 der ganzen Länge voneinander frei sind, und der Staubfaden ist mit seinem verdünnten Ende an dem Staubbeutel befestigt, und zwar an der Stelle, wo beide Pollensäcke am unteren Ende voneinander frei zu werden beginnen. Wenn der Staubbeutel reift, öffnen sich beide Pollensäcke durch Längsspalten und streuen eine reichliche Menge der kugeligen, glatten Pollenkörner aus. Aus dem oberen Ende des Fruchtknotens gehen die an ihren Spitzen die federförmige Narbe tragenden Griffel hervor. Bezüglich der Zahl der Griffel nahmen manche Autoren zwei an, welche sich gegenüberstehen, während nach meiner eigenen Beobachtung an meiner Sorte nicht zwei, sondern drei Griffel zu sehen sind. Frellich bietet es in den meisten Fällen den Anschein, als ob nur zwei Griffel vorhanden seien, allein wenn man genauer untersucht, so wird man leicht finden, daß außer diesen zwei noch ein schuppenförmiger rudi-

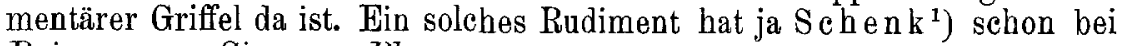
Brizopyrum, Sicurum, Phragmites, Calamagrostis, Aira und Lamarckia beobachtet. Bei unserer Sorte findet man außerdem nicht selten Blüten mit drei vollausgebildeten Griffeln. Ich beobachtete in den Jahren 1908 und 1909150 Blüten im ganzen; von denen 7 mit drei vollausgebildeten Griffeln, 142 mit zwei und 1 sogar mit vier derselben versehen waren. Im Jahre 1910 beobachtete ich auch viele hundert Blüten und fand wiederum einige mit drei wohlausgebildeten Griffeln: gelegentlick auch bei den Blüten mit zwei Griffeln ist einer derselben nahe seiner Basis zu zwei verzweigt, so daß man ohne genaue Beobachtung solche leicht für drei Griffel ansehen könnte. Dieser Fall ist leicht von demjenigen zu unterscheiden, wo wirklich drei Griffel zu sehen sind, da man im ersten Falle außer zwei Griffeln stets noch einen rudimentären findet. Die scheinbar viergriffelige Blüte ist durch die Verzweigung jedes der zwei vollausgebildeten Griffel nahe ihrer Basis entstanden. Im Jahre 1910 schrieb auch J. Schuster ${ }^{2}$ ) über die Rudimente eines Griffels in der Reisblüte, was mit meiner oben angeführten Beobachtung im Einklang steht. Aus der obigen Darstellung geht zweifellos hervor, daß die Reisblüte in ihrer Urform drei Griffel gehabt hat, deren einer meistens zu einem kleinen Rudiment reduziert ist.

Was die Lage der Griffel beirifft, so befindet sich einer gegen den Mittelnerv der Vorspelzen und die zwei andern richten sich nach der Deckspelze, zueinander im Winkel von ungefähr $120^{\circ}$ liegend. Der

1) Schenk, Untersuchungen des Baues der Grasblüte. Verhandl. naturw. Ver. Preul. Rheinlande, 1867.

2) J. Schuster, Über die Morphologie der Reisblüte. Flora, 1910, C. 
rudimentäre Griffel steht immer gegen den Mittelnerv der Vorspelze. Auch in der Blüte mit zwei Griffeln stehen sie sich nicht gerade gegenuber, sondern richten sich mebr oder weniger schräg nach den Deckspelzen. Der Winkel zwischen diesen zwei Griffeln ist variabel: in einem Falle beträgt er $120^{\circ}$, wie wir es in der
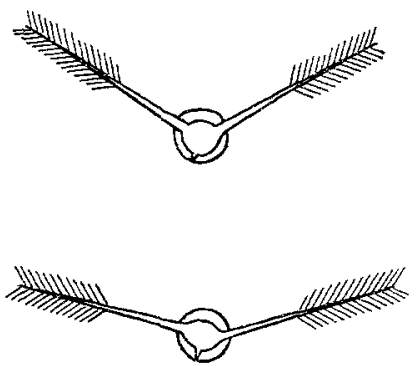

Abb.3. Flächenansicht von Pistillen mit zwei vollausgebildeten Griffeln. Blüte mit drei Griffeln sehen, im anderen ist er größer, fast $180^{\circ}$. Zu bemerken ist noch, dafo der rudimentäre Griffel nicht durch die Mißbildung eines anfangs normalen Griffels entsteht, sondern er ist schon als solcher sichtbar, wenn die Länge der Ähre noch kaum $3 \mathrm{~mm}$ ist, d. h. vom Anfang ihrer Entwieklung an. Nun will ich auf das Blütendiagramm des Reises zu sprechen kommen. Meines Wissens haben Eichler ${ }^{1}$ ) und $\mathrm{Hackel}^{2}$ ) es angegeben, und auch einige japanische Autoren. Nach meiner Ansichtbedürfen alle diese einer Berichtigung, denn sie nahmen die Zahl der Griffel als zwei an, oder erwähnten sie gar nicht. Das von mir aufgestellte Diagramm ist in Abb. 4 angegeben.

Wie aus dem Gesagten hervorgeht, gibt die Reisblüte, zusammen mit der von Streptochaeta und Bambusa Belege für die Ánsicht, daß das

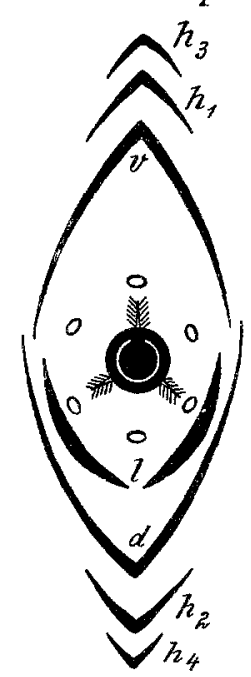

Abb. 4. Diagramm der Reisblüte nach der Ansicht desVerfassers. $d$ Deckspelze, $v$ Vorspelze, 7 Lodiculae, $h_{1} h_{2}$ $h_{3} h_{4}$ Hüllspelzen.

1) Eichler, Blütendiagramme, I, 1875. II, 2, 1887 . besteht.
Pistill der Gramineen ursprünglich dreizählig, d.h. daß das Gynäceum trikarpellär gewesen ist. Diese Tatsache und das hexamere Andröceum der Reisblüte bestätigt ferner die Ansicht, dab der phylogenetisch primitive Typus der Gramineen ein zweizyklisches Perigon bat, von dem jeder Zyklus aus drei getrennten Blättern

Ferner will ich über die Infloreszenz des Reises einige Mitteilungen machen. Es ist eine allgemein angunommene Tatsache, daß die Inflorescenz des Reises eine sogenannte "Rispe“ ist. Der Ausdruck "Rispe" ist leider keine genau bestimmte Bezeichnung, wie schon Linné, Gray, Wettstein, Warming - Johannsenu.a. geäußert haben. Er ist nur eine allgemeine Bezeichnung für die pyramidenförmig zusammengesetzte Infloreszenz, und wird manchmal als eine rein racemöse Infloreszenz angenommen (Sachs, Karsten). Freilich gehören mehrere Rispen zum racemösen Typus, was sich durch eine akropetale, resp. zentripetale Aufblühfolge und Nichtübergipfelung der obersten Blüten kennzeichnet. Bei einigen Rispen verbält es sich aber ganz anders, und solches ist gerade beim Reis der Fall. Wir sehen beim Reis eine basipetale Aufblühfolge und die Übergipfelung der Endblüte. Ich konnte feststellen, daß hier

Gramineae in Engler und Prantl, Natürl. Pflanzenfam., 
die Blüten am oberen Teile der Rispe zuerst entstanden sind und dann die Blüten am Unterteile in ihrer Entwicklung folgen. Dies geschieht nicht allein in der ganzen Rispe, sondern in gleicher Weise an den einzelnen Seitenachsen; so ist hier also die Übergipfelung der Endblüte die Regel.

Ich habe weiter versucht, die Aufbluhfolge zu bestimmen. Zunäcbst gilt es hier, die Morphologie der Reisinfloreszenz kurz zu schildern. Die Reisinfloreszenz hat eine verlängerte Hauptachse, von welcher bald wechselständig, bald gegenständig, bald auch wirtelständig mehrere Seitenachsen abzweigen, welche wiederum 1-3 sekundäre Seitenachsen nahe ihrer Basis produzieren; an jeder von diesen letzten Achsen sitzt je eine gestielte Blüte.

Wenn die äußeren Bedingungen günstig sind, wird sich das Aufblühen in folgender Weise vollziehen:

1. Die zuerst aufblühenden sind die Blüten der Seitenachsen in der Nähe der Ährenspitze, d. h. die erste bis vierte Seitenachse sind die Vorläufer des Auf blühens.

2. In der einzelnen Seitenachse sind die Vorläufer einige am Gipfelende oder diesem zunächst sitzende Blüten.

3. Das Gesagte gilt auch für das Verhältnis zwischen der Seitenachse und der sekundären Seitenachse, d. h. das Auf blühen in der Seitenachse schreitet von der sekundären Seitenachse neben

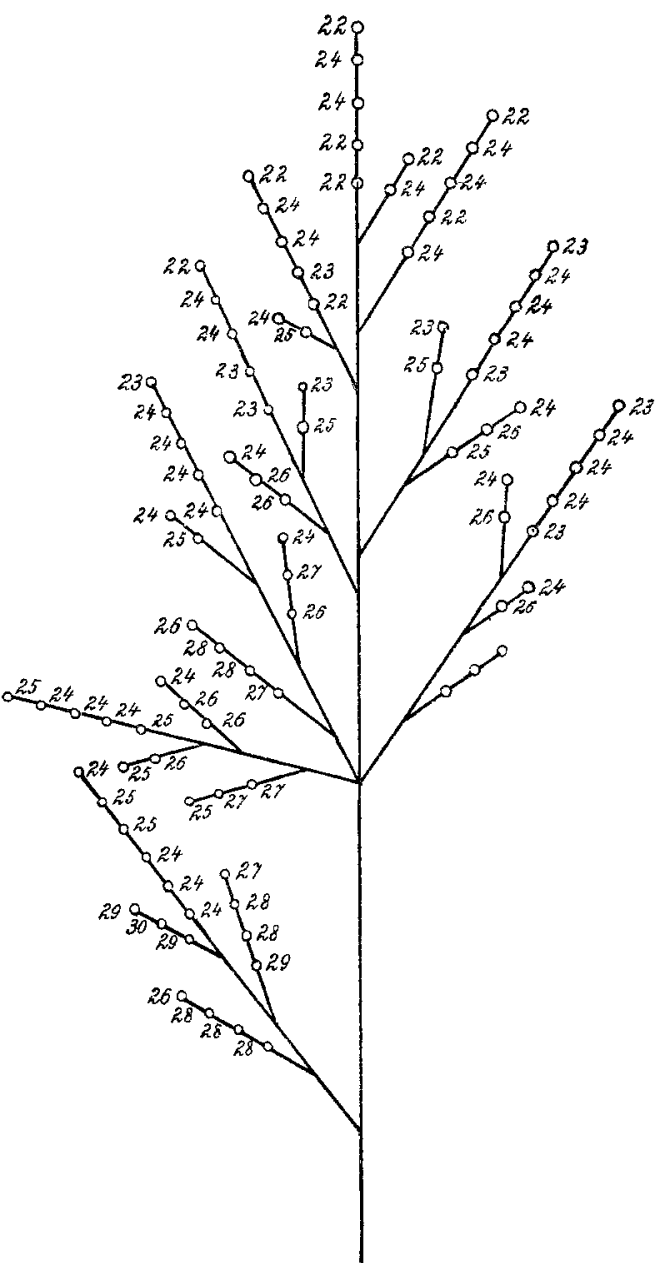

Abb. 5. Beispiel der Aufblühfolge einer Reisrispe. der Spitze allmählich nach der Basis zu, und in einer sekundären Seitenacbse auch in derselben Folge.

4. Der Zeitraum, in welchem das Aufblühen in einer Seitenachse sich vollzieht, beträgt meistens 3-4 Tage, öfters 7 Tage, da die Seitenachsen in dem unteren Teile der Ähre mehr sekundäre Seitenachsen tragen als im oberen, so für ihr ganzes Aufblühen längere Zeit benötigend.

5. Der Zeitraum, in welchem die ganze Ähre ihr Aufblühen vollendet, beträgt gewöhnlich 7 Tage, öfters 9 Tage oder sogar mehr. 
Um die eben erwähnten Tatsachen klar zu machen, will ich hier ein Beispiel meiner Beobachtung über die Aufblührolge angeben (Abb. 5). Das Bild repräsentiert eine Rispe, in welcher die Blüten so bezeichnet sind, daß die beigefügte Ziffer den Tag anzeigt, an welchem die betreffende Blüte sich geöffnet hat.

Aus den oben ausgeführten zwei Gründen, nämlich der Entwicklungsgeschichte und der Autblühfolge, geht zweifelsohne hervor, das die Reisinfloreszenz basipetal oder cymös gebaut ist, daß daher die Annahme, dab ihre Rispe racemös sei. nicht richtig ist.

Den Herren Prof. Dr. S. Ikeno, Prof. Dr. N. Ohno und Dr. N. Yats u bin ich für ihre gütige Unterstützung zu großjem Danke verpflichtet.

\title{
Phylogenetische Studien über die Gattung Monoclea.
}

\author{
(Mit 1 Textabbildung.) \\ Von Viktor Schiffner (Wien).
}

(Schluf. ${ }^{1}$ )

\section{Das männliche Receptaculum.}

Für Campbell und Johnson ist eine der wichtigsten Stützen ilhrer Ansicht, daß Monoclea zu den Marchantiales gehöre, die B $\theta-$ schaffenheit des o Receptaculums: "There is no structure closely comparable with the male receptacle of Monoclea known among the Jungermanniaceae" (Johnson. l. c., p. 199). Dies ist aber unrichtig, denn wenn man auch die großse Übereinstimmung in der äußeren Form und im Bau mit den analogen Organen gewisser Marchantiales (Plagiochasma, Reboulia etc.) zugeben muß, so gibt es doch auch unter den Jangermaniaceae ein Analogon dafür, $\mathrm{u}$. $\mathrm{zw}$. ist es die auch habituell und in vielen Details mit Monoclea so sebr übereinstimmende Gattung Makinoa, die hier in Betracht kommt, welche auch sonst merkwürdigerweise von Campbell und Johnson vollkommen ignoriert wird.

Wie bei Monoclea besitzt auch Makinoa scharf u mschriebene Antheridienstände ( $\varangle$ Receptakeln) auf der Fronsoberseite und dies ist sonst bei keiner anderen Gattung der Jungermaniaceae der Fall. Ein Blick anf die Abbildungen von Miyake (Hedw.. 1899, Tab. IX, F'ig. 5, 6, und Tokyo Bot. Magaz., 1899, Tab. III), die nach meinen Untersuchungen eines reichlichen Originalmateriales sehr treffend sind, zeigt sofort die prinzipielle Übereinstimmung. Die Receptakeln sind scharf begrenzte Gruppen von in die Fronsoberseite eingesenkten Antheridien wie bei Monoclea, nur in der Form weichen sie etwas von diesen ab, da es nicht erbabene Polster sind, die auch gegen den Fronsscheitel zu scharf abfallen, sondern nur nach rückwärts scharfrandig und jäh abfallend sind, wodurch sie von oben gesehen fast halbmondförmig erscheinen. Die Intensität der Teilungen (der. Zellwucherung) der die Antheridienanlagen umgebenden Fronszellen, aus der die Versenkung der Antheridien resultiert, ist bei Monoclea eine viel größere als bei Makinoa und aus dieser rein

1) Vgl. Nr. 1, S. $29-33_{2}$ Nr. 2, S. 75-81, Nr. 3, S. 113-121. 